
2 Research Square
Preprints are preliminary reports that have not undergone peer review.
They should not be considered conclusive, used to inform clinical practice,
or referenced by the media as validated information.

\title{
Growth Hormone Supplementation During Ovarian Stimulation Does Not Alter Outcomes In Non-Poor Ovarian Response Patients With Multiple IVF Failures
}

\author{
Jingwei Yang \\ Chongqing Key Laboratory of Human Embryo Engineering \\ Xiaodong Zhang \\ Chongqing Key Laboratory of Human Embryo Engineering \\ Guo Jing \\ Chongqing Key Laboratory of Human Embryo Engineering \\ Qiaoli Chen \\ Chongqing Key Laboratory of Human Embryo Engineering \\ Guoning Huang ( $\square$ gnhuang217@sina.com ) \\ Chongqing Key Laboratory of Human Embryo Engineering
}

\section{Research Article}

Keywords: Growth hormone, Non-poor ovarian response patients, Multiple IVF failures

Posted Date: February 3rd, 2022

DOI: https://doi.org/10.21203/rs.3.rs-1256203/v1

License: (c) (1) This work is licensed under a Creative Commons Attribution 4.0 International License. Read Full License 


\section{Abstract}

Background: Growth hormone (GH) is being used in different clinical situations in assisted reproductive technologies (ART), but its role has yet to be determined. The aim of this study was to determine the effect of human growth hormone (GH) supplementation during ovarian stimulation in IVF cycles on non-poor ovarian response (non-POR) patients showing two previous failures of IVF cycles with no clearly identifiable reasons.

Method: This is a retrospective cohort study of 139 women who underwent a $3^{\text {rd }}$ IVF cycle with adjuvant GH treatment $(n=52)$, compared retrospectively to patients also received a $3^{\text {rd }}$ IVF treatment but without $\mathrm{GH}$ during the same period of time $(n=87)$. Generalized estimating equation (GEE) models were used to evaluate the relation between $\mathrm{GH}$ and the cumulative clinical pregnancy rate (CCPR) or the number of available embryos.

Results: With multiple cycles the proportion of $\mathrm{GnRH}$-ant was higher in the $\mathrm{GH}$ group than $\mathrm{GH}$-free group (GH group vs.GH-free: $1^{\text {st }}$ cycle, $11.54 \%$ vs. $8.04 \% ; 2^{\text {nd }}$ cycle, $44.23 \%$ vs. $33.33 \% ; 3^{\text {rd }}$ cycle, $69.23 \%$ vs. $\left.59.77 \% ; P<0.001\right)$. The CCPR of the $3^{\text {rd }}$ cycle was highest in self-paired cycles (GH


was higher than that of the $\mathrm{GH}$ group in the $3^{\text {rd }} \mathrm{cycle}(40.23 \% \mathrm{vs} .36 .54 \%, P<0.01)$. The use of fixed-effects GEE models revealed that after adjusting for potential confounders, the application of $\mathrm{GH}$ was not significantly associated with an increase in the CCPR or the number of available embryos $(\mathrm{OR}=1.05[95 \% \mathrm{Cl}, 0.46-2.47]$ and $0.98[95 \% \mathrm{Cl}, 0.66-1.48] ; P>0.05)$.

Conclusions: The data obtained herein indicated that GH co-treatment in IVF cycles did not exert any obvious effect on non-POR patients.

\section{Background}

Growth hormone $(\mathrm{GH})$ is being used in different clinical situations in assisted reproductive technologies (ART)-including poor ovarian response, polycystic ovary syndrome (PCOS), and poor oocyte or embryo quality [1-3]. Numerous investigators have over the past 30 years used GH supplementation during ovarian stimulation for in vitro fertilization (IVF) cycles, but its role in IVF has yet to be determined.

$\mathrm{GH}$ is produced by the anterior pituitary gland and is hypothesized to exhibit several modes of action during follicular development. Even though a pivotal role for GH has not yet been determined, other co-effectors such as insulin-like growth factor-I (IGF-I) and leukemia inhibitory factor (LIF) cannot be neglected due to their roles in the ovary in augmenting steroidogenesis and oocyte maturation[4-5]. GH allows full maturation of denuded human oocytes[6-8], and its receptor is present in cumulus cells and in the human oocyte [8]. The signaling of GH is two-fold: in addition to IGF-1, GH also utilizes the Janus kinase-signal transducer and activator of transcription (JAK-STAT)-mediated pathway [3]. Large prospective studies and randomized controlled trials (RCTs) of GH co-treatment in IVF cycles have been published in recent years, and many IVF centers worldwide still use various add-ons without knowledge of their true beneficial effects or cost-effectiveness. Mounting evidence suggests that GH supplementation is one of the few interventions that may potentially improve IVF success rates [9].

The majority of clinical studies on adjuvant GH have focused on patients with poor ovarian response (POR), and there have been several studies that have shown significantly improved ovarian response and embryonic parameters [9-11]. A recent meta-analysis of the use of GH in IVF on poor responders [12-13] showed that there was a positive benefit on the number of oocytes retrieved, number of oocytes fertilized, and the clinical pregnancy rate, but that there was no difference in live-birth rate. Other authors noted that the use of $\mathrm{GH}$ in other types of ovarian responders has yet to be investigated [14]. A study on adjuvant GH in IVF previously showed that there was no benefit in ovarian response, embryo quality, or pregnancy rate in normal responders [15]. And for normal responders who underwent failed IVF treatment 2 or more times, the probability of improving oocyte/embryo quality for embryo implantation and development with $\mathrm{GH}$ co-treatment remains unclear.

Therefore, we aimed to compile data from clinical studies with the use of a rigorous methodology to offer evidence of the impact of $\mathrm{GH}$ addition on the outcome of a third IVF cycle in non-poor responders who failed two or more previous IVF treatments.

\section{Methods}

This was a retrospective study in which we linked patient information from EMRs in the Chongqing Health Center for Women and Children database to evaluate women with non-poor ovarian response (> 3 oocytes retrieved) who started their first IVF treatment under the age of 38 , and who had experienced three cycles of ovum-pick up (OPU) (including IVF and intracytoplasmic sperm injection [ICSI]) from January of 2016 to December of 2019. Oocyte quality and the likelihood of pregnancy and live birth in ART diminishes with increasing age in women, and drops markedly after 40 years of age [16-18]. The 139 patients included in this study underwent their IVF cycles with either a GnRH-a or GnRH-ant protocol. Exclusion criteria were as follows. Patients with PCOS, those whose oocytes were vitrified, whose oocyte retrieval was canceled, and those patients undergoing preimplantation genetic testing (PGT) cycles, or manifesting chromosomal abnormalities or uterine malformations were excluded. At the third OPU cycle, the patients treated with $\mathrm{GH}(\mathrm{n}=52)$ were compared with the $\mathrm{GH}$-free group $(\mathrm{n}=87)$. Importantly, all patients showed failure in their two previous IVF cycles (Fig. 1).

Patients in the GH group received three IU of recombinant human GH (Jintropin AQ, Gensci, Changchun, China) per day, from the initial day of downregulation using the long protocol or stimulation using the antagonist protocol until the day of the hCG trigger. The average duration of GH cotreatment was 30 days for patients receiving the long agonist protocol and 10 days for those receiving the antagonist protocol.

Page 2/11 


\section{Stimulation Protocol}

\section{GnRH-agonist long protocol}

GnRH-a (triptorelin, $0.1 \mathrm{mg} / \mathrm{d}$ or $0.05 \mathrm{mg} / \mathrm{d}$, sc.; Decapeptyl, Ferring, Germany) was used for pituitary downregulation starting in the previous luteal phase. After administration of GnRH agonist for 14-21 days-and when the levels of estrogen were $<50 \mathrm{pg} / \mathrm{mL}$, luteinizing hormone $<5 \mathrm{mIU} / \mathrm{mL}$, and $\mathrm{P}<1 \mathrm{ng} / \mathrm{mL}-$ a dose of recombinant follicle-stimulating hormone ( $\mathrm{rFSH}$ ) at a dose ranging from 75 to $300 \mathrm{IU}$ was then administrated subcutaneously per day based on the woman's age, anti-Müllerian hormone (AMH) level, and antral follicle count (AFC).

GnRH-antagonist protocol: Controlled ovarian stimulation (COS) was initiated on day 2 or 3 of the cycle at a dose of rFSH ranging from 75 to 300 IU. The GnRH antagonist ( 0.25 mg; Orgalutran, Organon, The Netherlands or Cetrorelix, Merck Serono, Switzerland) was given to patients daily if at least one of the following criteria was fulfilled: (i) the presence of at least one follicle $>14 \mathrm{~mm}$ in diameter, (ii) a serum estrogen level of $>600 \mathrm{pg} / \mathrm{mL}$, and (iii) a serum LH level of $>10 \mathrm{IU} / \mathrm{L}$ (11).

\section{Trigger Day}

When at least three follicles measured $>18 \mathrm{~mm}$ in diameter, human chorionic gonadotropin (hCG, Merck Serono, Italy) was administered, and some women undergoing $\mathrm{GnRH}$-ant cycles received triptorelin acetate injection ( $\mathrm{GnRH}-\mathrm{a}$, Ferring $\mathrm{GmbH}$, Germany) as trigger. Transvaginal oocyte retrieval was performed $36 \mathrm{~h}$ later, and then embryo transfer (ET) on day 3 post-oocyte retrieval. Luteal-phase support was initiated immediately after oocyte retrieval by combined vaginal and oral progesterone. Surplus viable embryos or all-frozen embryos (used due to the potential for ovarian hyperstimulation syndrome [OHSS], or a thin endometrium, abnormal blood biochemical index, or other personal situations of patients) were frozen for later transfer in subsequent frozen-embryo transfer (FET) cycles. The vast majority of these embryos were frozen on day 3 . Embryos that were not suitable for cryopreservation on day 3 were cultured until day 5 or 6 and vitrified if they reached the blastocyst stage. Luteal-phase support with combined vaginal-oral progesterone was started 3 days before FET.

\section{Vitrification And Storage}

The Cryotop vitrification method was performed according to the manufacturer's instructions and as reported by Kuwayama et al. [19], and the Kitazato kit (Kita, Toyota, Japan) was used for vitrification. Embryos were equilibrated in VS1 solution for 5 min before exposure to vitrification solution (VS 2). The embryos were dipped in vitrification solution (VS 2) for $30 \mathrm{~s}$, and the vitrification procedure was executed according to the manufacturer's protocol. Embryos were then placed on a Cryotop sheet and excess vitrification solution was removed by aspiration using a pipette prior to the immersion of the carrier in LN2.

\section{Warming Procedure}

A Dewar flask of LN2 containing the carriers was placed close to the microscope. We used forceps to grasp the straw in the LN2 and place it in a dish containing $3 \mathrm{~mL}$ of $1.0 \mathrm{~mol} / \mathrm{L}$ sucrose at $37^{\circ} \mathrm{C}$ for $1 \mathrm{~min}$. All embryos were transferred sequentially to 0.5 and $0.25 \mathrm{~mol} / \mathrm{L}$ sucrose solutions at room temperature for 3 min each. The embryos were then washed several times in Quinn's 1024 (Cooper Surgical, CT, USA) solution and placed in G1 medium (Vitrolife, Kungsbacka, Sweden) for further culture. Post-warming survival of cryopreserved embryos was defined as the survival of more than one-half of the original cells that were intact.

\section{Outcome Measures}

The indices of embryonic quality were the day 2-4 cell (D2-4c) rate and day 3-8 cell (D3-8c) rate, defined as the proportion of embryos with $4 / 8$ cells on day $2 / 3$, respectively, of the total number of two-pronuclear (2PN)-stage embryos. The primary outcome was the number of available embryos and cumulative clinical pregnancy rate (CCPR) per OPU, the latter defined as the presence of a fetal sac during ultrasonographic examination at 6 weeks and which resulted from an ART cycle-including fresh and FETs that resulted from the associated ovarian stimulation. If a pregnancy occurred, then the patients were said to have obtained an outcome, regardless of subsequent cycles.

\section{Statistics}

Data are presented as means (SD) or number (\%), as appropriate. We used the Wilcoxon rank-sum test to analyze continuous variables and a nonparametric paired test was used for self-paired variables. The CCPRs in the GH and GH-free groups were compared using Chi-squared test. The generalized estimating equation (GEE) models were used to evaluate the relative prognostic significance of treatment groups, women's age, protocol, the ovarian sensitivity index (OSI=[number of retrieved oocytes/total gonadotropin dose] $\times 1000$ ), insemination and primary cause of infertility in 
relation to CCPR, and the number of available embryos. We adjusted interactions between independent covariates. $P$-values of $<0.05$ were considered to indicate statistical significance. All analyses were conducted using Stata (version 15.1, College Station, TX; StataCorp. LLC).

\section{Results}

A total of 139 non-POR patients who underwent treatment with $\mathrm{GH}(\mathrm{n}=52$ ) were compared with the $\mathrm{GH}$-free group ( $\mathrm{n}=87$ ), and all patients exhibited two previous IVF cycle failures. The demographic data and cycle characteristics of self-paired cycles are summarized in Table 1. In multiple cycles the proportion of $\mathrm{GnRH}$-ant use was increased overall in the groups, and was higher in the $\mathrm{GH}$ group relative to the $\mathrm{GH}$-free group (GH group vs. $\mathrm{GH}$-free group: 1 st cycle, $11.54 \%$ vs. $8.04 \%$; 2 nd cycle, $44.23 \%$ vs. $33.33 \%$; 3 rd cycle, $69.23 \%$ vs. $59.77 \%$, respectively; $P<0.001$ ). And in the multiple cycles, the proportion of women undergoing ICSI was increased overall in the groups, with the ICSI proportion higher in the $\mathrm{GH}$ group than in the GH-free group in the third cycle (48.08\% vs. $41.38 \%, P<0.01)$. There was no difference between the groups in terms of age, infertility during, and concentrations of $\mathrm{AMH}, \mathrm{FSH}, \mathrm{E2}$, LH and number of AFC in both groups were not different in multiple cycles (GH group vs. GH-free group, $8.47 \pm 3.89$ vs. $9.04 \pm 4.26$; $8.84 \pm 3.23$ vs. $8.42 \pm 4.22 ; 8.85 \pm 3.2$ vs. $8.61 \pm 3.3$, respectively; $P>0.05)$.

Table 1

Demographic data and cycle characteristics of self-paired cycles.

\begin{tabular}{|lllllll|}
\hline & 1st cycle & & 2nd cycle & & 3rd cycle \\
\hline & GH & GH-free & GH & GH-free & GH & GH-free \\
\hline Age, years & $32.29 \pm 3.84$ & $32.37 \pm 4.62$ & $33.17 \pm 3.84$ & $33.35 \pm 4.64$ & $33.94 \pm 3.83$ & $34.11 \pm 4.74$ \\
\hline Infertility during & $5.38 \pm 3.8$ & $5.14 \pm 4.16$ & $5.62 \pm 3.82$ & $5.58 \pm 4.15$ & $6.25 \pm 3.93$ & $6.37 \pm 4.47$ \\
\hline AMH(ng/mL) & $3.07 \pm 2.75$ & $3.10 \pm 2.18$ & $2.93 \pm 2.22$ & $2.88 \pm 2.08$ & $2.87 \pm 1.83$ & $3.00 \pm 2.41$ \\
\hline FSH(IU/L) & $5.84 \pm 1.2$ & $6.06 \pm 1.27$ & $5.46 \pm 1.24$ & $5.8 \pm 1.51$ & $5.63 \pm 1.3$ & $5.75 \pm 1.43$ \\
\hline E2(pg/ml) & $34.25 \pm 17.5$ & $39.28 \pm 18.56$ & $35.14 \pm 16.26$ & $35.72 \pm 18.46$ & $33.93 \pm 15.14$ & $32.79 \pm 14.95$ \\
\hline LH(IU/L) & $2.99 \pm 1.28$ & $3.07 \pm 1.76$ & $2.94 \pm 1.2$ & $3.04 \pm 1.79$ & $2.93 \pm 1$ & $3.04 \pm 1.23$ \\
\hline AFC (n) & $8.47 \pm 3.46$ & $9.04 \pm 4.02$ & $8.84 \pm 3.23$ & $8.42 \pm 3.22$ & $8.85 \pm 3.2$ & $8.61 \pm 3.3$ \\
\hline GnRH-a protocol & $46 / 52(88.46)^{\mathrm{a}}$ & $80 / 87(91.95)^{\mathrm{b}}$ & $29 / 52(55.77)^{\mathrm{a}}$ & $58 / 87(66.67)^{\mathrm{b}}$ & $16 / 52(30.77)^{\mathrm{a}}$ & $35 / 87(40.23)^{\mathrm{b}}$ \\
\hline GnRH-ant protocol & $6 / 52(11.54)^{\mathrm{a}}$ & $7 / 87(8.04)^{\mathrm{b}}$ & $23 / 52(44.23)^{\mathrm{a}}$ & $29 / 87(33.33)^{\mathrm{b}}$ & $36 / 52(69.23)^{\mathrm{a}}$ & $52 / 87(59.77)^{\mathrm{b}}$ \\
\hline IVF(\%) & $42 / 52(80.77)$ & $69 / 87(79.31)$ & $30 / 52(57.69)$ & $52 / 87(59.77)$ & $27 / 52(51.92)^{\mathrm{a}}$ & $51 / 87(58.62)^{\mathrm{b}}$ \\
\hline ICSI(\%) & $10 / 52(19.23)$ & $18 / 87(20.69)$ & $22 / 52(42.31)$ & $35 / 87(40.23)$ & $25 / 52(48.08)^{\mathrm{a}}$ & $36 / 87(41.38)^{\mathrm{b}}$ \\
\hline a vs. ${ }^{\mathrm{b}}, P<0.05$ in each cycle. & & & & & \\
\hline
\end{tabular}

However, in multiple cycles, there was no significant difference on concentrations of AMH, FSH, E2, LH, OSI, or ET endometrium between groups or between self-paired cycles $(P>0.05)$. Based on the same OSI, there was a significant increase in the number of available embryos between self-paired cycles (GH group, $1.87 \pm 1.33$ vs. $1.62 \pm 1.46$ vs. $2.25 \pm 1.73$, respectively; $P=0.015$, GH-free group: $1.93 \pm 1.54$ vs. $1.83 \pm 1.4$ vs. $2.83 \pm 1.81, P=$ 0.001 ) and between the groups in the 3 rd cycle (GH group vs GH-free group, $2.25 \pm 1.73$ vs. $2.83 \pm 1.81$, respectively, $P=0.01$ ) (Table 2, Fig. 2 ). 
Table 2

cos results of multiple cycles.

\begin{tabular}{|c|c|c|c|c|c|c|}
\hline & \multicolumn{2}{|l|}{ 1st cycle } & \multicolumn{2}{|l|}{ 2nd cycle } & \multicolumn{2}{|l|}{ 3rd cycle } \\
\hline & $\mathrm{GH}$ & GH-free & $\mathrm{GH}$ & GH-free & $\mathrm{GH}$ & GH-free \\
\hline $\begin{array}{l}\text { E2 on HCG day } \\
(\mathrm{pg} / \mathrm{ml})\end{array}$ & $2830.82 \pm 1398.41$ & $3061.15 \pm 1302.16$ & $2751.17 \pm 1385.78$ & $2582.85 \pm 1472.03$ & $2040.58 \pm 1100.35$ & $2298.18 \pm 1290.09$ \\
\hline $\begin{array}{l}\text { FSH on HCG } \\
\text { day }(I \mathrm{IU} / \mathrm{L})\end{array}$ & $13 \pm 5.49$ & $14.55 \pm 6.73$ & $12.59 \pm 3.84$ & $13.22 \pm 5.04$ & $12.62 \pm 4.36$ & $13.92 \pm 4.66$ \\
\hline $\begin{array}{l}\text { LH on HCG } \\
\text { day(IU/L) }\end{array}$ & $1.31 \pm 0.7$ & $1.4 \pm 1.76$ & $1.24 \pm 0.79$ & $1.34 \pm 0.85$ & $1.86 \pm 2.15$ & $1.68 \pm 1.38$ \\
\hline $\begin{array}{l}\text { HCG } \\
\text { endometrium(mm) }\end{array}$ & $9.62 \pm 1.83$ & $9.72 \pm 1.38$ & $8.89 \pm 1.52$ & $9.4 \pm 1.56$ & $9.06 \pm 1.75$ & $9.25 \pm 1.58$ \\
\hline Gn day & $10.43 \pm 1.51$ & $10.35 \pm 2.19$ & $9.84 \pm 1.72$ & $9.9 \pm 2.17$ & $9.54 \pm 1.97$ & $9.81 \pm 2.08$ \\
\hline Gn dose (IU) & $2330.39 \pm 773.78$ & $2382.5 \pm 838.49$ & $2275.5 \pm 569.43$ & $2371.19 \pm 765.51$ & $2376.06 \pm 849.04$ & $2413.7 \pm 708.19$ \\
\hline ET endometrium & $1.04 \pm 0.18$ & $0.99 \pm 0.16$ & $0.97 \pm 0.15$ & $1.02 \pm 0.19$ & $0.99 \pm 0.17$ & $1.02 \pm 0.16$ \\
\hline $\begin{array}{l}\text { Retrieval of } \\
\text { oocyte(n) }\end{array}$ & $9.65 \pm 6.02$ & $9.09 \pm 4.95$ & $10.49 \pm 5.15$ & $8.74 \pm 4.64$ & $9.08 \pm 4.78$ & $9.26 \pm 4.66$ \\
\hline OSI & $4.79 \pm 3.73$ & $4.12 \pm 2.68$ & $4.9 \pm 2.92$ & $4.01 \pm 2.83$ & $4.62 \pm 3.4$ & $4.35 \pm 3.09$ \\
\hline $\begin{array}{l}\text { Available } \\
\text { embryos(n) }\end{array}$ & $1.87 \pm 1.33^{\mathrm{a}}$ & $1.93 \pm 1.54^{b}$ & $1.62 \pm 1.46^{\mathrm{a}}$ & $1.83 \pm 1.4^{b}$ & $2.25 \pm 1.73^{a}$ & $2.83 \pm 1.81^{b}$ \\
\hline \multicolumn{7}{|c|}{${ }^{\mathrm{a}} P=0.015$ in self-paired cycle (GH group). } \\
\hline 001 in & ( $\mathrm{GH}$ fro & & & & & \\
\hline
\end{tabular}

With respect to estimating the quality of the oocyte and embryo, we observed significant difference between groups in the normal fertilization rate and polyspermy rate of the 3rd cycle (GH group vs. GH-free group, $58.47 \%$ vs. $65.22 \% ; 5.93 \%$ vs. 9.09\%, respectively; $P<0.05$ ). The D2-4c and D3-8c rates of the 2nd cycle were significantly different between groups (GH group vs. GH-free group, $45.58 \%$ vs. $54.01 \%$; $21.55 \%$ vs. $37.73 \%$, respectively; all $P$ values $<0.05$ ). The CCPR of the 3rd cycle was highest in self-paired cycles (GH group, 30.77\% vs., $25.00 \%$ vs., $36.54 \%$; GH-free group, $32.18 \%$ vs. $28.74 \%$ vs. $40.23 \%$, respectively; all $P$ values $<0.001$ ), and the CCPR of the $\mathrm{GH}$-free group was elevated relative to the $\mathrm{GH}$ group in the 3 rd cycle $(40.23 \%$ vs. $36.54 \%, P<0.01)$. There was no significant difference in the $\mathrm{M} 2$ oocyte rate or $2 \mathrm{PN}$ cleavage rate of embryos between groups, or in the self-paired cycles of either group $(P>0.05)$ (Table 3$)$. 
Table 3

Embryo quality of patients treated in multiple cycles.

\begin{tabular}{|c|c|c|c|c|}
\hline & & 1 st cycle & 2nd cycle & 3rd cycle \\
\hline \multirow[t]{2}{*}{ M II oocyte rate } & $\mathrm{GH}$ & $402 / 492$ (81.71) & 405/493 (82.15) & $379 / 472$ (80.30) \\
\hline & GH-free & $572 / 691$ (82.78) & $570 / 664(85.84)$ & 630/759 (83.02) \\
\hline \multirow[t]{2}{*}{ Normal fertilization rate } & $\mathrm{GH}$ & 277/492(56.30) & 298/493(60.45) & $276 / 472(58.47)^{\star}$ \\
\hline & GH-free & 411/691(59.48) & $406 / 664(61.14)$ & $495 / 759(65.22)^{*}$ \\
\hline \multirow[t]{2}{*}{ Polyspermy rate } & $\mathrm{GH}$ & 49/492(9.96) & 44/493(8.92) & $28 / 472(5.93)^{\star}$ \\
\hline & $\mathrm{GH}$-free & 71/691(10.27) & 64/664(9.64) & $69 / 759(9.09) *$ \\
\hline \multirow[t]{2}{*}{ 2PN cleavage rate } & $\mathrm{GH}$ & 266/277(96.03) & 283/298(94.97) & 253/264(95.83) \\
\hline & $\mathrm{GH}$-free & $388 / 411(94.40)$ & 387/406(95.32) & 480/495(96.97) \\
\hline \multirow[t]{2}{*}{ D2-4c rate } & $\mathrm{GH}$ & $144 / 266(54.14)$ & $129 / 283(45.58) \star$ & 131/253(51.78) \\
\hline & $\mathrm{GH}$-free & 191/388(49.23) & $209 / 387(54.01) *$ & $262 / 480(54.58)$ \\
\hline \multirow[t]{2}{*}{ D3-8c rate } & $\mathrm{GH}$ & $76 / 266(28.57)$ & $61 / 283(21.55)^{\star}$ & 75/253(29.64) \\
\hline & $\mathrm{GH}$-free & 112/388(28.87) & $146 / 387(37.73) \star$ & 172/480(35.83) \\
\hline \multirow[t]{2}{*}{ CCPR } & $\mathrm{GH}$ & $16 / 52(30.77)^{a}$ & $13 / 52(25.00)^{a}$ & $19 / 52(36.54)^{a}$ \\
\hline & GH-free & $28 / 87(32.18)^{b}$ & $25 / 87(28.74)^{b}$ & $35 / 87(40.23)^{b}$ \\
\hline \multicolumn{5}{|c|}{$\star P<0.01$ between groups (GH group vs. GH-free group) } \\
\hline \multicolumn{5}{|c|}{${ }^{\mathrm{a}} P=0.002$ in self-paired cycles (GH group) } \\
\hline
\end{tabular}

Fixed-effects GEE models revealed that after adjusting for potential confounders (age, protocol, OSI, insemination), the application of GH exhibited no significant association with increased CCPR or number of available embryos (OR $=1.05$ [95\% $\mathrm{Cl}, 0.46-2.47$ ] and 0.98 [95\% Cl, 0.66-1.48], respectively; $P>0.05$ ) (Table 4$)$.

Table 4

Adjusted associations between treatment and CCPR/available embryos.

\begin{tabular}{|lllll|}
\hline Independent variable & \multicolumn{2}{l}{ CCPR } & & \multicolumn{2}{l|}{ EMBRYOS } \\
\cline { 2 - 5 } Dependent variable & OR & $95 \% \mathrm{Cl}$ & OR & $95 \% \mathrm{Cl}$ \\
\hline Treatment (with GH/without GH) & 1.05 & $0.46-2.47$ & 0.98 & $0.66-1.48$ \\
\hline Protocol (GnRH-a/GnRH-ant) & 0.89 & $0.66-1.20$ & $1.16 *$ & $1.01-1.35$ \\
\hline Age & 0.98 & $0.93-1.04$ & $1.07 *$ & $1.04-1.10$ \\
\hline OSI & 1.01 & $0.92-1.11$ & $1.2 *$ & $1.11-1.29$ \\
\hline IVF/ICSI & 0.93 & $0.51-1.71$ & 0.82 & $0.61-1.09$ \\
\hline Available embryos & $1.49 *$ & $1.21-1.82$ & $/$ & $/$ \\
\hline Fertilization & $/$ & $/$ & $1.02 *$ & $1.01-1.03$ \\
\hline$* P<0.05$ & & & & \\
\hline
\end{tabular}

\section{Discussion}


GH has been used as an adjuvant in ovarian stimulation for over 25 years [10]; however, its place in ART and its influence are yet to be clarified. Many investigators using $\mathrm{GH}$ have studied women with a poor ovarian response, leading to the collection of a greater number of oocytes relative to women who received treatments without $\mathrm{GH}$, and many of the early clinical parameters appeared favorable [20-22]. Whether a role for $\mathrm{GH}$ lies in the treatment of poor oocyte quality or in a subset of poor-responder patients, its position in treating non-POR patients awaits further investigation and clarification.

The present investigation was a strictly controlled retrospective study, and to the best of our knowledge, we are the first to examine the impact of GH co-treatment during multiple IVF/ICSI cycles in non-POR patients. We demonstrated that using GH in addition to gonadotropins in recurrent assistedcycle failures did not improve clinical outcomes in non-POR patients. In the GH group, the number of available embryos and the CCPR in the third IVF cycle were much higher than in two previous failures in self-paired controls, but were closer to that observed for the comparative group undergoing no $\mathrm{GH}$ co-treatment. After adjusting for confounders, we showed that the positive changes in self-paired controls were not related to $\mathrm{GH}$ co-treatment.

Considering our study outcomes, it remains controversial as to the efficacy of GH. The population of the present study consisted of non-POR patients who showed normo-ovarian reserve, and our results were based on a previous study that showed that in normo-ovulatory women (under 38 years of age) the addition of $\mathrm{GH}$ to a long $\mathrm{GnRH}-\mathrm{a} / \mathrm{hMG}$ protocol did not improve ovarian response, and that $\mathrm{GH}$ supplementation did not result in an increase in either serum E2 levels, number of follicles, oocytes, or embryos [15]. But studies have shown inconsistent results in different populations. One study showed that the use of GH in IVF cycles where PGT-A was performed improved the ploidy of embryos in patients who were not classified as having a POR [23]. And women aged $>40$ years undergoing ART and co-stimulated with $\mathrm{GH}$ achieved more ongoing pregnancies and underwent less pregnancy wastage, more deliveries, and more live births [21].

In our study, patients may have undergone a modified treatment protocol after failure of the previous IVF cycle, and the proportions receiving GnRH-a and $\mathrm{GnRH}$-ant were different between the $\mathrm{GH}$ and $\mathrm{GH}$-free groups. Multiple studies-including meta-analyses and randomized controlled trials (RCTs) -on the effects of $\mathrm{GnRH}$-a and $\mathrm{GnRH}$-ant protocols on pregnancy rate and live-birth rate have yielded controversial findings [24-25]. Due to the differences in normal fertilization rate and the number of available embryos (endometrial sensitivity remained unchanged) that we observed in our results, asynchronization of follicular development [26] and decreased endometrial receptivity with the GnRH-ant protocol might still be related to the clinical outcomes [27-28]. Using the OSI is quite suitable for incorporation into more complex prediction models of IVF outcome, and OSI is significantly correlated with the currently used biomarkers of ovarian responsiveness, is more predictive of clinical pregnancy than the total number of oocytes, and is highly consistent in repeated IVF cycles even when the COS protocol changes [29]. To attenuate the polyspermy rate, the mode of insemination is partially changed from IVF to ICSI in the patient's next cycle. In the GEE model, OSI is an impactful factor with respect to available embryo number, but IVF/ICSI is not a crucial factor for available embryos or CCPR.

Results from human and animal studies have demonstrated that GH stimulates the production of insulin-like growth factor 1 (IGF-1) in serum and from ovarian follicles [30]. In animal models, IGF-1 exerts an inhibitory effect on follicular apoptosis, and the avoidance of apoptosis is essential for follicular development and oocyte maturation [31-32]- suggesting that GH promotes follicular development and the inhibition of follicular apoptosis via IGF-1. And previous investigators have also reported that reproductive aging leads to a decline in the number of functionally viable mitochondria in human oocytes, and that the mitochondrial activity in oocytes diminishes with age [33-34]. GH as an adjunct to standard ovarian stimulation significantly increased oocyte mitochondrial function in POR patients [35], and studies have confirmed that the interactions with cumulus granulosa cells (CGCs) ensure that oocytes possess enough energy production in reserve for meiosis and to support subsequent embryonic development during oocyte maturation [36]. The mtDNA content in CGCs is positively associated with embryo development and implantation competence, and is a reliable biomarker for the assessment of oocyte quality [37]. However, these aforementioned studies focused on the effects of GH on POR patients, and there exists no large dataset of CGC mtDNA copy number for the assessment of GH actions on oocytes in non-POR patients.

There were several limitations to our study-one being the study design, which was retrospective in nature; however, we still provided preliminary evidence to support further prospective investigations into the role of adjuvant GH in women who are non-POR. Another limitation was our sample size, which was relatively small due to few patients having undergone multiple IVF cycles and meeting our exclusion criteria. Nevertheless, we demonstrated that GH co-treatment in IVF/ICSI cycles wielded no obvious effect on non-POR patients. These results remain to be confirmed by a large-scale, randomized controlled trial.

\section{Abbreviations}

ART, assisted reproductive technology

COS, controlled ovarian stimulation

$\mathrm{GH}$, Growth hormone

IVF, in vitro fertilization

GEE, Generalized estimating equation 
POR, poor ovarian response

LIF,leukemia inhibitory factor

IGF-I,insulin-like growth factor-I

ICSI,intracytoplasmic sperm injection

ET,embryo transfer

FET,frozen-embryo transfer

OHSS,ovarian hyperstimulation syndrome

CCPR,cumulative clinical pregnancy rate

AMH,anti-Müllerian hormone

AFC,antral follicle count

rFSH,recombinant follicle-stimulating hormone

OPU,ovum-pick up

OSI,ovarian sensitivity index

\section{Declarations}

\section{Ethics approval and consent to participate}

The study involving human participants was reviewed and approved by the Ethics Committee Review Board of Chongqing Health Center for Women and Children (approval number: 2018-RGI-04) for retrospective analysis and clinical data reporting. In accordance with the measures of the People's Republic of China on the administration of Human Assisted Reproductive Technology, the ethical principles of the Human Assisted Reproductive Technology as well as the Helsinki declaration. Informed consent was waived by the Ethics Committee Review Board of Chongqing Health Center for Women and Children because of the retrospective nature of the study.

\section{Consent for publication}

Not applicable

\section{Availability of data and materials}

The datasets used and analyzed in the current study are not publicly available due to personal information of patients but are available from the corresponding author on reasonable request.

\section{Competing interests}

The authors declare no competing interests.

\section{Funding}

This study was supported by a grant from the Medical Research Foundation of Chongqing to Qiao-Li Chen (grant number 2020MSXM90).

\section{Authors' contributions}

The present work was designed by GH. Data extraction and analysis were performed by JY and XZ. JY and JG participated in the data collection. GH, QC participated in revisions to the article. All authors have read and approved the final manuscript.

\section{Acknowledgements}

Not applicable

\section{References}

1. Gong Y, Luo S, Fan P, et al. Growth hormone alleviates oxidative stress and improves oocyte quality in Chinese women with polycystic ovary syndrome: a randomized controlled trial. Scientific Reports. 2020; 10(1): 18769. doi: 10.1038/s41598-020-75107-4. 
2. Hazout A, Junca A M, Y Ménézo, et al. Effect of growth hormone on oocyte competence in patients with multiple IVF failures. Reproductive Biomedicine Online. 2009; 18(5):664-670.

3. Huang Z H, Baxter R C, Hughes S M, et al. Supplementary growth hormone treatment of women with poor ovarian response to exogenous gonadotrophins: changes in serum and follicular fluid insulin-like growth factor-1 (IGF-1) and IGF binding protein-3 (IGFBP-3). Human Reproduction. 1993; 8 (6):850-7. doi:10.1093/oxfordjournals.humrep.a138153

4. Ptak G, F Lopes, Matsukawa K, et al. Leukaemia inhibitory factor enhances sheep fertilization in vitro via an influence on the oocyte. Theriogenology. 2006; 65(9):1891-1899. doi: 10.1016/j.theriogenology.2005.10.018

5. Ipsa E, Cruzat V F, Kagize J N, et al. Growth Hormone and Insulin-Like Growth Factor Action in Reproductive Tissues. Frontiers in Endocrinology. 2019; 10:777. doi: 10.3389/fendo.2019.00777

6. Hassan H A, Azab H, Rahman A A, et al. Clinical Assisted Reproduction: Effects of Growth Hormone on in Vitro Maturation of Germinal Vesicle of Human Oocytes Retrieved from Small Antral Follicles. Journal of Assisted Reproduction \& Genetics. 2001; 18(8):417-420. doi:10.1023/a:1016630603560

7. Menezo, Y. J., Nicollet, B., Rollet, J., \& Hazout, A. Pregnancy and delivery after in vitro maturation of naked ICSI-GV oocytes with GH and transfer of a frozen thawed blastocyst: case report. J Assist Reprod Genet. 2006; 23(1): 47-49. doi: 10.1007/s10815-005-9014-0

8. Menezo Y, Nicollet B, Rollet J, et al. Pregnancy and delivery after in vitro maturation of naked ICSI GV oocytes with GH and transfer of a frozen thawed blastocyst: case report. Journal of Assisted Reproduction \& Genetics. 2006; 23(1):47-49. doi: 10.1017/s096719940300234x

9. Jeve Y B, Bhandari H M. Effective treatment protocol for poor ovarian response: A systematic review and meta-analysis. Journal of Human Reproductive Sciences. 2016; 9(2):70-81. doi: 10.4103/0974-1208.183515

10. Hart R J, Rombauts L, Norman R J. Growth hormone in IVF cycles: any hope? Curr Opin Obstet Gynecol. 2017;29(3): 119-125. doi: 10.1097/GCO.0000000000000360

11. Kolibianakis E M, Venetis C A, Diedrich K, et al. Addition of growth hormone to gonadotrophins in ovarian stimulation of poor responders treated by in-vitro fertilization: a systematic review and meta-analysis. Human Reproduction Update. 2009; (6):613-622. doi: 10.1093/humupd/dmp026

12. Eftekhar M, Aflatoonian A, et al. Adjuvant growth hormone therapy in antagonist protocol in poor responders undergoing assisted reproductive technology. Archives of Gynecology and Obstetrics. 2013; 287(5):1017-1021.doi: 10.1007/s00404-012-2655-1.

13. Bayoumi Y A, Dakhly D, Bassiouny $Y$ A, et al. Addition of growth hormone to the microflare stimulation protocol among women with poor ovarian response. International Journal of Gynecology \& Obstetrics. 2015; 131(3): 305-308. doi: 10.1016/j.ijgo.2015.05.034

14. Polyzos, N. P., \& Sunkara, S. K. Sub-optimal responders following controlled ovarian stimulation: an overlooked group? Hum Reprod. 2015; 30(9): 2005-2008. doi: 10.1093/humrep/dev149

15. Younis J S, Simon A, Koren R, et al. The effect of growth hormone supplementation on in vitro fertilization outcome: a prospective randomized placebo-controlled double-blind study. Fertility \& Sterility. 1992; 58(3):575-580. doi: 10.1016/s0015-0282(16)55266-2

16. Hull M, Fleming C F, Hughes A O, et al. The age-related decline in female fecundity: A quantitative controlled study of implanting capacity and survival of individual embryos after in vitro fertilization. Fertility and Sterility. 1996; 65(4):783-790.

17. Janny L, Menezo Y. Maternal age effect on early human embryonic development and blastocyst formation. Molecular Reproduction and Development. 1996; 45(1):31-37.

18. DE Battaglia, Goodwin P, Klein N A, et al. Influence of maternal age on meiotic spindle assembly in oocytes from naturally cycling women. Human Reproduction. 1996; 11(10):2217-2222.

19. Chian R C, Kuwayama M, Tan L, et al. High survival rate of bovine oocytes matured in vitro following vitrification. J Reprod Dev. 2005; 50(6):685696. doi: $10.1262 / \mathrm{jrd} .50 .685$

20. Gong Y, Zhang K, Xiong D, et al. Growth hormone alleviates oxidative stress and improves the IVF outcomes of poor ovarian responders: A randomized controlled trial. 2020; 18(1): 91. doi: 10.1186/s12958-020-00648-2

21. Jan T, H André, Carmen M. Improvement of delivery and live birth rates after ICSI in women aged $>40$ years by ovarian co-stimulation with growth hormone. Human Reproduction. 2005; 20(9): 2536-2541. doi: 10.1093/humrep/dei066.

22. Yovich J L, Ye Y, Keane K N. Growth Hormone Adjuvant trial for poor responders undergoing IVF. European Journal of Obstetrics \& Gynecology and Reproductive Biology. 2019; 236: 249. doi: 10.1016/j.ejogrb.2019.01.026.

23. Skillern A, Leonard W, Pike J, et al. Growth hormone supplementation during ovarian stimulation improves oocyte and embryo outcomes in IVF/PGT-A cycles of women who are not poor responders. Journal of Assisted Reproduction and Genetics. 2021;1-6.doi: 10.1007/s10815-02102088-2

24. Alinany H G, Youssef M A, Ayeleke R O, et al. Gonadotrophin-releasing hormone antagonists for assisted reproductive technology. Fertility \& Sterility. 2011; 96(3):S261-S261. doi: 10.1002/14651858.CD001750.pub4

25. Al-Inany, Hesham G. Cochrane Database of Systematic Reviews (Reviews). Gonadotrophin-releasing hormone antagonists for assisted reproductive technology.1996. doi:10.1002/14651858.cd001750.pub4

26. Lin P Y, Huang F J, Fu-Tsai K, et al. Reassessing the feasibility of the zygote score for predicting embryo viability in IVF/ICSI using the GnRH antagonist protocol compared to the long protocol. Plos One. 2017; 12(2): e0171465. doi: 10.1371/journal.pone.0171465

Page 9/11 
27. Bahçeci M, Ulug U, Erden HF, et al. Frozen-thawed cleavage-stage embryo transfer cycles after previous GnRH agonist or antagonist stimulation. Reproductive Biomedicine Online. 2009; 18(1):67-72. doi:10.1016/s1472-6483(10)60426-9

28. Rackow B W, Kliman H J, Taylor H S. GnRH antagonists may affect endometrial receptivity. Fertility \& Sterility. 2008; 89(5):1234-1239. doi: 10.1016/j.fertnstert.2007.04.060

29. Revelli A, Gennarelli G, Biasoni V, Benedetto C, et al. The Ovarian Sensitivity Index (OSI) Significantly Correlates with Ovarian Reserve Biomarkers, Is More Predictive of Clinical Pregnancy than the Total Number of Oocytes, and Is Consistent in Consecutive IVF Cycles. J Clin Med. 2020; 9(6). doi: $10.3390 / \mathrm{jcm} 9061914$

30. S, Pellegrini, B, et al. Physiology: In-vivo studies on ovarian insulin-like growth factor I concentrations in human preovulatory follicles and human ovarian circulation. Hum. Reprod. 1995;10(6):1341-1345. doi:10.1093/humrep/10.6.1341

31. Chun S Y, Billig H, Tilly J L, et al. Gonadotropin suppression of apoptosis in cultured preovulatory follicles: mediatory role of endogenous insulinlike growth factor I. Endocrinology. 1994; (5):1845. doi:10.1210/endo.135.5.7525255

32. Baker J, Hardy M P, Zhou J, et al. Effects of an Igf1 gene null mutation on mouse reproduction. Molecular Endocrinology. 1996; 10(7):903. DOl: 10.1210/me.10.7.903

33. Elpida, Fragouli, Katharina, et al. Altered Levels of Mitochondrial DNA Are Associated with Female Age, Aneuploidy, and Provide an Independent Measure of Embryonic Implantation Potential. Plos Genetics. 2015; 11(6): e1005241. doi: 10.1371/journal.pgen.1005241

34. Sheng $X$, Yang $Y$, Zhou J, et al. Mitochondrial transfer from aged adipose-derived stem cells does not improve the quality of aged oocytes in C57BL/6 mice: SHENG et al. Molecular Reproduction and Development. 2019; 86(5): 516-529. doi: 10.1002/mrd.23129

35. Weall B M, Al-Samerria S, Conceicao J, et al. A direct action for GH in improvement of oocyte quality in poor-responder patients. Reproduction. 2015; 149(2): 147-154. doi: 10.1530/REP-14-0494

36. A, Taugourdeau, V, et al. The mitochondrial DNA content of cumulus cells may help predict embryo implantation. Journal of Assisted Reproduction \& Genetics. 2018; 36(2): 223-228. doi: 10.1007/s10815-018-1348-5

37. Li J, Chen Q, Wang J, et al. Does growth hormone supplementation improve oocyte competence and IVF outcomes in patients with poor embryonic development? A randomized controlled trial. BMC Pregnancy and Childbirth. 2020; 20(1):310. doi: 10.1186/s12884-020-03004-9

\section{Figures}



Figure 1 
Flow chart. GH, growth hormone; OPU, ovum-pick up; PCOS, polycystic ovary syndrome; PGT, preimplantation genetic testing; CCPR, cumulative clinical pregnancy rate.
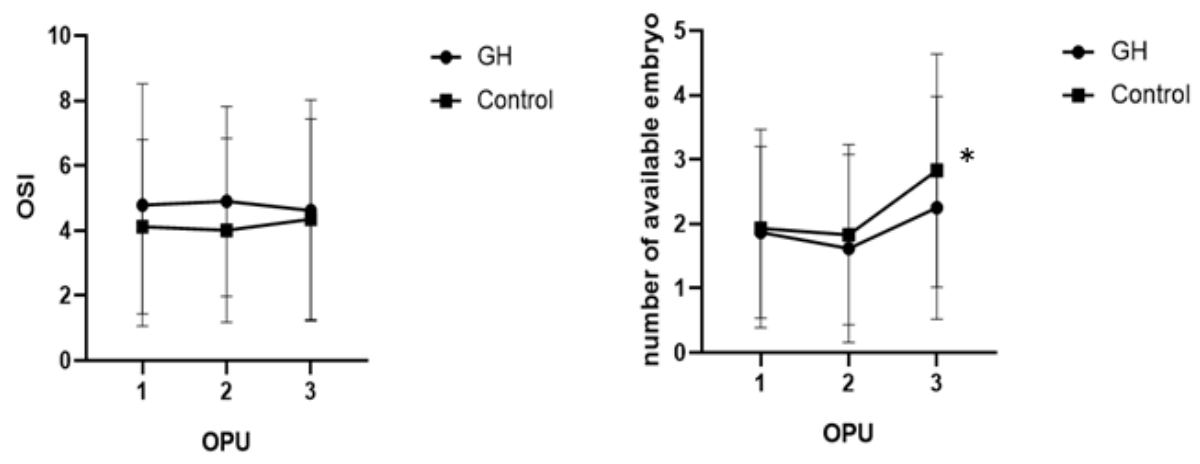

Figure 2

The changes in $\mathrm{OSI} /$ the number of available embryos across multiple cycles. 